\title{
Evaluation of Retinal Function and Flicker Light-Induced Retinal Vascular Response in Normotensive Patients with Diabetes without Retinopathy
}

\author{
Amélie Lecleire-Collet, ${ }^{1}$ Isabelle Audo, ${ }^{2}$ Mounir Aout ${ }^{3}$ Jean-François Girmens, ${ }^{2}$ \\ Rénata Sofroni, ${ }^{1}$ Ali Erginay, ${ }^{1}$ Jean-François Le Gargasson, ${ }^{2}$ Saddek Moband-Saïd, ${ }^{2}$ \\ Taly Meas, ${ }^{4}$ Pierre-Jean Guillausseau, ${ }^{4}$ Eric Vicaut,${ }^{3}$ Michel Paques, ${ }^{2}$ and Pascale Massin ${ }^{1,2}$
}

Purpose. To correlate retinal function with vascular response to flicker light in normotensive patients with diabetes without diabetic retinopathy (DR).

Methods. Twenty-eight normotensive patients with diabetes (11 with type 1, 17 with type 2) without DR and 28 sex- and age-matched healthy control subjects underwent color vision and contrast sensitivity testing, pattern, full-field, and multifocal electroretinography, and evaluation of the vascular response to flicker light with the dynamic vessel analyzer.

RESULTs. In the patients with diabetes, electroretinogram (ERG) pattern responses, $b$-wave in the scotopic bright flash ERG, $a$-wave and $b$-wave in the photopic single-flash ERG, and oscillatory potential responses were significantly impaired compared with those in control subjects. Vascular response to flicker light was also impaired in patients with diabetes compared with controls. In the whole population, correlations were found between flicker light-induced arterial retinal vasodilation and the amplitude and implicit time of the N95 wave of pattern ERG $(r=-0.27, P=0.047$ and $r=-0.35, P=$ 0.008 , respectively), the $b$-wave implicit time of $\operatorname{rod}$ ERG $(r=$ $-0.36 ; P=0.01)$ and the oscillatory potentials $(r=0.4 ; P=$ 0.003 ), suggesting that impairment of the vascular response to flicker light may reflect inner retinal neural impairment. However, no correlation between these factors was found when only patients with diabetes were considered.

Conclusions. In patients with diabetes, neural and neurovascular dysfunctions both precede the onset of clinically detectable DR. To which extent these abnormalities are related to each other remains to be determined. (ClinicalTrials.gov number,

From the Departments of ${ }^{1}$ Ophthalmology and ${ }^{4}$ Internal Medicine B, Hôpital Lariboisière, and the ${ }^{3}$ Clinical Research Unit, Hôpital Fernand Widal, Assistance Publique-Hôpitaux de Paris, Paris 7 University, Paris, France; and the ${ }^{2}$ Center for Clinical Investigation 503 INSERM, Centre Hospitalier National d'Ophtalmologie des XV-XX, Pierre et Marie Curie, Paris 6 University, Paris, France.

Supported by Grant CIRC CRC04148, PO41009 from the Délégation à la Recherche Clinique de l'Assistance Publique-Hôpitaux de Paris, France.

Submitted for publication May 26, 2010; revised October 17 and November 13, 2010; accepted December 5, 2010.

Disclosure: A. Lecleire-Collet, None; I. Audo, None; M. Aout None; J.-F. Girmens, None; R. Sofroni, None; A. Erginay, None; J.-F. Le Gargasson, None; S. Mohand-Saïd, None; T. Meas, None; P.-J. Guillausseau, None; E. Vicaut, None; M. Paques, None; P. Massin, None

Corresponding author: Pascale Massin, Department of Ophthalmology, Hôpital Lariboisière, 2 rue Ambroise Paré, 75475 Paris Cedex 10, France; p.massin@lrb.aphp.fr.
NCT00839150.) (Invest Ophthalmol Vis Sci. 2011;52:2861-2867) DOI:10.1167/iovs.10-5960

$\mathrm{D}$ iabetic retinopathy (DR) is classically considered a form of microangiopathy, although recent data suggest that early abnormalities in DR may be caused by neuroglial cell impairment. ${ }^{1,2}$ Several authors have reported that impairment of neurovascular coupling in the retina of patients with diabetes precedes clinically visible DR. ${ }^{3-9}$ However, these authors were unable to reach the definitive conclusion that primary neural or vascular damage was at the origin of these abnormalities because the patients were not tested for retinal neural function. Moreover, some series have included patients with diabetes and various levels of DR, associated arterial hypertension, or both, ${ }^{3-6,8,9}$ which might have affected the results. ${ }^{4-6,8-10}$ Therefore, to improve understanding of the early retinal events preceding the onset of DR, we combined, in a group of normotensive patients with diabetes but no DR, an analysis of neural and neurovascular function using retinal neural functional tests and the dynamic vessel analyzer (DVA) with flicker light stimulation.

\section{Patients and Methods}

\section{Patients}

The study adhered to the tenets of the Declaration of Helsinki. Written informed consent was obtained from the participants after they had been given oral and written explanations. The study was approved by the local ethics committee.

Inclusion criteria for patients with diabetes were age between 20 and 55 years, type 1 or type 2 diabetes mellitus, diabetes duration of $>5$ years, no clinically detectable DR on dilated indirect funduscopy, and retinal color photographs according to the Early Treatment of Diabetic Retinopathy Study criteria.

Inclusion criteria for sex- and age-matched control subjects were no history of high blood sugar and a fasting blood sugar level below $1.10 \mathrm{~g} / \mathrm{L}$

Exclusion criteria for both patients and controls were the presence of cataract (defined as lens opacities assessed with the Lens Opacities Classification System III equal to or more than nuclear opalescence stage 2, nuclear color stage 2, cortical opacities stage 2 , or posterior subcapsular opacities stage 2), or history of cataract surgery, history of glaucoma, intraocular pressure of $>21 \mathrm{~mm} \mathrm{Hg}$ using Goldmann applanation tonometry, abnormal score on Ishihara 24-plate test, or any other ocular disease. Patients with ametropia of $>3$ diopters, anisometropia, or astigmatism of $>1$ diopter were excluded. Also excluded were patients with untreated hypertension (defined as systolic blood pressure $>140 \mathrm{~mm} \mathrm{Hg}$ and diastolic blood pressure $>90 \mathrm{~mm} \mathrm{Hg}$ ) or treated hypertension, those smoking $>20$ cigarettes a day, and those taking vasoactive drugs, alcohol, or drugs adversely affecting color 
vision. All subjects underwent complete ocular examination. A complete history of diabetes and glycated hemoglobin (A1C) measurements was recorded for each patient with diabetes.

\section{Study Protocol}

All participants were asked to refrain from alcohol, caffeine, and tobacco consumption for 12 hours before DVA examination. Blood pressure measurements and glycemia were assessed at the beginning of the study. For all participants, the right eye was the one assessed.

\section{Color Vision}

Color vision was tested with the desaturated 15 Hue test (D-15DS; Lanthony, Luneau, France), and the results were expressed as the total color difference score (TCDS) ${ }^{11}$ and as the number of abnormal transpositional crossovers ( $\geq 2$ color caps) compared with each confusion axis (type 1 , type 2 , and type 3 , the equivalents of protan, deutan, tritan, and tetartan axes for hereditary color disorders).

\section{Contrast Sensitivity}

Subjects were tested with the Pelli-Robson contrast sensitivity chart (Haag- Streit UK, Essex, UK) using the recommended conditions.

\section{Electroretinography}

Pattern and full-field electroretinograms (ERGs; Vision Monitor, Métrovision, Pérenchies, France) and multifocal ERGs (Veris, EDI Inc., San Mateo, CA) were recorded for each patient, according to the International Society for Clinical Electrophysiology of Vision (ISCEV) protocol. ${ }^{12-14}$

For pattern ERG, recordings were performed binocularly to facilitate optimal fixation. Gold foil corneal recording electrodes were placed on the tested eyes, and both the reference and the ground electrodes were placed in the outer canthus and the center of the forehead, respectively.

A black-and-white reverse checkerboard was used; the aspect ratio of the width over the height of the stimulus field did not exceed 4:3. The mean width and height of the stimulus field was 158 , with a check size of 0.88 . The photopic luminance level for the white areas was greater than $80 \mathrm{~cd} / \mathrm{m}^{2}$. The contrast between black and white squares was close to $100 \%$. The frame rate of the CRT had a frequency of $75 \mathrm{~Hz}$. The reversal rate was $2.2 \mathrm{~Hz}$. Typically, $>100$ sweeps were averaged by using "interrupted stimulation" to minimize eye movement artifacts. Patients were instructed to concentrate on the stimulus for 4 to 5 seconds without blinking; averaging was then suspended, and the patient was told to blink before again, concentrating on the fixation spot.

Cone responses were recorded as part of the full-field ERG after recording responses under scotopic conditions. Full-field ERG was recorded according to the ISCEV standard published in 2004. Neither angiography nor retinal imaging was performed before recordings. Pupils were optimally dilated, and both active and ground electrodes were placed in the same way as for the recording of multifocal and pattern ERGs. Recording was performed binocularly. Before the measurements, patients remained in darkness for 20 minutes. Rod-specific responses were recorded in response to a dim flash attenuated from $2.5 \mathrm{log}$ units of the standard flash. A stimulus using a standard flash of $1.5 \mathrm{~cd} \cdot \mathrm{s} / \mathrm{m}^{2}$ was used to record the standard combined ERG under dark-adapted conditions. The patient was then light adapted, using a background light of $17 \mathrm{~cd} / \mathrm{m}^{2}$ within the full-field stimulus bowl for 10 minutes. Responses to the standard flash were then recorded both after single stimulation and a $30-\mathrm{Hz}$ period.

Oscillatory potential analysis was performed according to the method of Yonemura. ${ }^{15}$ The peak implicit time of the first deflection of the oscillatory potential, the interpeak interval between the first and second deflections, and the sum of the amplitudes of the upward deflections were precisely measured with a ruler. ${ }^{16}$
For multifocal (mf) ERG recordings, the recording and ground electrodes were placed as they were for the recording of pattern ERG. The other eye was occluded. An array of 103 hexagons across a field subtending $44^{\circ}$ horizontally and $40^{\circ}$ vertically, scaled by eccentricity, was displayed at a frame rate of $75 \mathrm{~Hz}$. Responses were bandpass filtered outside 10 to $300 \mathrm{~Hz}$, amplified at a gain of 100,000, and sampled every $0.833 \mathrm{~ms}$. A standard $m$-sequence length was used, with $m=15$, resulting in a total recording time of 7.17 minutes divided into 16 short segments for patients' comfort. Signals were amplified (gain, $\left.10^{6}\right)$, bandpass filtered $(10-100 \mathrm{~Hz})$, and recorded with a sampling interval of $0.83 \mathrm{~ms}\left(16 \times\right.$ per video game). Luminance was $200 \mathrm{~cd} / \mathrm{m}^{2}$ for the white symbol and $\leq 2 \mathrm{~cd} / \mathrm{m}^{2}$ for the black symbol. The surrounding luminance was set at $50 \%$ of the bright test luminance (i.e., $100 \mathrm{~cd} / \mathrm{m}^{2}$ ). Standard ambient room lighting was used throughout the day of the study. Stimulus luminance was calibrated with an EDI grid calibrator (Veris; EDI Inc.).

Average responses for the implicit times and amplitudes of N1 (first negative component), P1 (first positive component), and N2 (second negative component) of the first-order kernel were calculated for three regional ring groups, defined as follows : ring 1 , from $0^{\circ}$ to $5^{\circ}$ eccentricity ( 7 central hexagons); ring 2 , from $5^{\circ}$ to $10^{\circ}$ eccentricity (30 hexagons), and ring 3 , from $10^{\circ}$ to $25^{\circ}$ eccentricity (66 hexagons).

\section{DVA Examination with Flicker Light Stimulation}

Before and during examination with the DVA, arterial blood pressure was monitored every 2 minutes. The DVA device and study protocol have been described elsewhere. ${ }^{4-6,8-10,17-19}$ To summarize, arterial and venous segments approximately $1.5 \mathrm{~mm}$ long were evaluated in each eye. Measurements were located 1 to 2 disc diameters from the optic disc. Selection criteria for the arterial and venous segment locations were as follows: no crossing or bifurcation in the measured segment, a curvature of not $>30^{\circ}$, a distance from the neighboring vessels of at least one vessel diameter, and sufficient contrast with the surrounding fundus. The standard program for flicker stimulation using DVA consists of three consecutive flicker cycles $(12.5-\mathrm{Hz}$ flicker frequency and 80-second observation each) and a total duration of the examination of 352 seconds. Three consecutive flicker cycles were therefore analyzed for each vessel segment studied. In healthy subjects, flicker stimulation leads to transient arterial vasodilatation followed by transient vasoconstriction and to transient venous vasodilatation. Response was measured as the difference between the mean vascular diameter for the last 10 seconds of flicker stimulation and the mean vascular diameter for the 30 seconds immediately preceding this flicker stimulation, divided by the latter value (Lecleire-Collet A, et al.

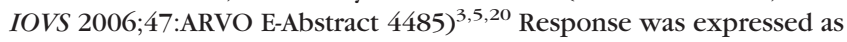
the mean of the calculations for the three flicker cycles. Only one artery and one vein were measured in each eye.

\section{Statistical Methods}

For the flicker-induced response, a generalized linear model was used that considered the factor diabetes versus control (taking into account the matching between diabetes and control) and vessel type (i.e., arterial or venous response). For the other parameters, either the paired Student's $t$-test or the Wilcoxon's signed rank test was used to compare control subjects and patients with diabetes. When a significant difference was found between patients with diabetes and controls, generalized linear models were used to compare patients with type 1 diabetes and type 2 diabetes, and each type of patient with diabetes with its corresponding matched control group. We checked that all the conditions of normality and homoscedasticity were fulfilled for all parametric statistical tests used. When normal assumptions were not met, rank transformations were used. Box plots (showing quartiles and range) were used for graphical presentation of the data.

Spearman correlation coefficient was used to calculate the correlations between individual variables. The significance level was set at $P<0.05$. Given the exploratory nature of the present study, no adjustment for multiplicity was made. All tests were performed using 
TABLE 1. Characteristics of Patients with Diabetes and Control Subjects

\begin{tabular}{lcccc}
\hline & Control & \multicolumn{2}{c}{ Patients with Diabetes } \\
\cline { 3 - 5 } \multicolumn{1}{c}{ Parameter } & $\begin{array}{c}\text { Subjects } \\
(\boldsymbol{n}=\mathbf{2 8})\end{array}$ & $\begin{array}{c}\text { Total } \\
(\boldsymbol{n}=\mathbf{2 8})\end{array}$ & $\begin{array}{c}\text { Type 1 } \\
(\boldsymbol{n}=\mathbf{1 1})\end{array}$ & $\begin{array}{c}\text { Type 2 } \\
(\boldsymbol{n}=\mathbf{1 7})\end{array}$ \\
\hline Age, y & $39.5 \pm 9.2$ & $39.9 \pm 9.4$ & $36.1 \pm 8.0$ & $42.4 \pm 9.6$ \\
Sex, male/female & $12 / 16$ & $12 / 16$ & $4 / 7$ & $8 / 9$ \\
Diabetes duration, y & $\mathrm{NA}$ & $12.4 \pm 7.8$ & $16.4 \pm 9.8$ & $9.8 \pm 5.0$ \\
Glycated hemoglobin (A1C), \% & $\mathrm{NA}$ & $7.8 \pm 1.5$ & $7.5 \pm 1.6$ & $8 \pm 1.4$ \\
Blood sugar, mmol/L & $4.9 \pm 0.6$ & $10.7 \pm 5.0^{*}$ & $10.3 \pm 6.0^{*}$ & $10.2 \pm 4.4^{*}$ \\
Mean systolic arterial pressure, mm Hg & $112.5 \pm 10.6$ & $118.8 \pm 14.4$ & $116.1 \pm 17.4$ & $120.6 \pm 12.3$ \\
Mean diastolic arterial pressure, mm Hg & $73.4 \pm 6.5$ & $77.5 \pm 8.3$ & $75.3 \pm 10.0$ & $79.0 \pm 7.0^{*}$ \\
Mean best-corrected visual acuity, & & & & \\
$\quad$ logMAR & $-0.02 \pm 0.09$ & $-0.05 \pm 0.12$ & $-0.04 \pm 0.10$ & $-0.06 \pm 0.13$ \\
Intraocular pressure, mm Hg & $14.8 \pm 1.8$ & $14.5 \pm 2.0$ & $14.4 \pm 1.4$ & $14.6 \pm 2.3$ \\
Baseline arterial vessel diameter, RU & $117.4 \pm 15.4$ & $122.7 \pm 25.2$ & $122.5 \pm 12.8$ & $122.8 \pm 31.5$ \\
Baseline venous vessel diameter, RU & $140.6 \pm 22.0$ & $151.8 \pm 21.0$ & $149.1 \pm 25.9$ & $153.6 \pm 17.6$ \\
\hline
\end{tabular}

Data are mean \pm SD. NA, not applicable; RU, relative units.

${ }^{*} P<0.05$ significant difference versus controls.

statistical software (SAS 9.2: SAS Institute, Cary, NC). Graphics were made using R software (cran.r-project.org).

\section{RESUlTS}

The characteristics of patients with diabetes and controls are given in Table 1 . Only the blood sugar level was significantly higher in the patients with diabetes than in the controls.

\section{Flicker-Induced Responses Measured Using the DVA}

Comparisons of vasodilations by general linear model demonstrated a significant difference between control subjects and diabetic patients both for arteries and for veins $(P<0.0001$; Fig. 1). No significant difference between arterial and venous responses $(P=0.12)$ and no significant interaction between factor "category of patients" and factor "vessel type" $(P=0.8)$ were observed.

\section{Color Vision}

The TCDS was significantly higher in patients with diabetes than in controls $(147.5 \pm 31.6$ vs. $130.1 \pm 14.9 ; P=0.02)$. The number of abnormal transpositional crossovers along the type 3 axis was also significantly higher in patients with diabetes (tritan axis: $0.30 \pm 0.54$ vs. $0.00 \pm 0.00, P=0.02$; tetar$\tan$ axis: $0.56 \pm 0.64$ vs. $0.07 \pm 0.26, P=0.003$ ). Neither TCDS nor abnormal transpositional crossovers along the type 3 axis differed significantly in patients with type 1 or type 2 diabetes, respectively, compared with controls or in patients with type 1 compared with type 2 diabetes.

\section{Contrast Sensitivity}

Contrast sensitivity scores were slightly lower in patients with diabetes than in controls $(1.64 \pm 0.08$ vs. $1.66 \pm 0.07)$, but the difference did not reach significance.

\section{Electroretinography}

Pattern ERG. The implicit time and amplitude of the P50 wave of patients with diabetes were significantly delayed and reduced $(P=0.01$ and $P=0.0001$, respectively); the implicit time and amplitude of the $\mathrm{N} 95$ wave were significantly delayed and increased $(P=0.0004$ and $P=0.003$, respectively, Fig. 2$)$. There was no significant difference between the pattern ERG of patients with type 1 diabetes and those with type 2 diabetes.

Full-Field ERG. For rod ERG, the implicit time and amplitude of the $b$-wave were significantly delayed and reduced in patients with diabetes compared with controls $(P=0.004$ and $P=0.02$, respectively; Fig. 3 ), but the implicit time and amplitude of the $a$-wave were not significantly different. For cone ERG, the implicit time of the $\boldsymbol{a}$ - and $b$-waves were significantly delayed in patients with diabetes compared with controls $(P=0.009$ and $P=0.01$, respectively $)$, and the amplitude of the $b$-wave was significantly reduced $(P=0.02)$. There was no significant difference between patients with type 1 diabetes and those with type 2 diabetes for either rod or cone ERG.

The 30-Hz flicker ERG exhibited trends toward reduced amplitudes and delayed implicit times, but neither trend was significant (data not shown).

The peak implicit time of the first deflection of the oscillatory potentials and the interpeak interval between the first and second deflections were significantly delayed in patients with
FIGURE 1. Flicker-induced response in retinal arteries (A) and veins (B) in patients with diabetes and control subjects.
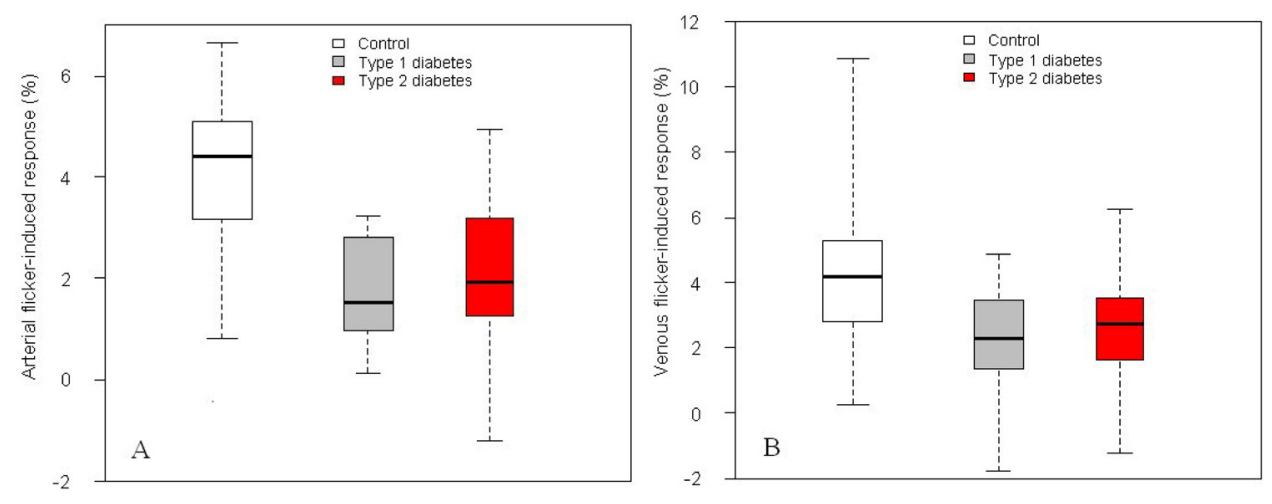

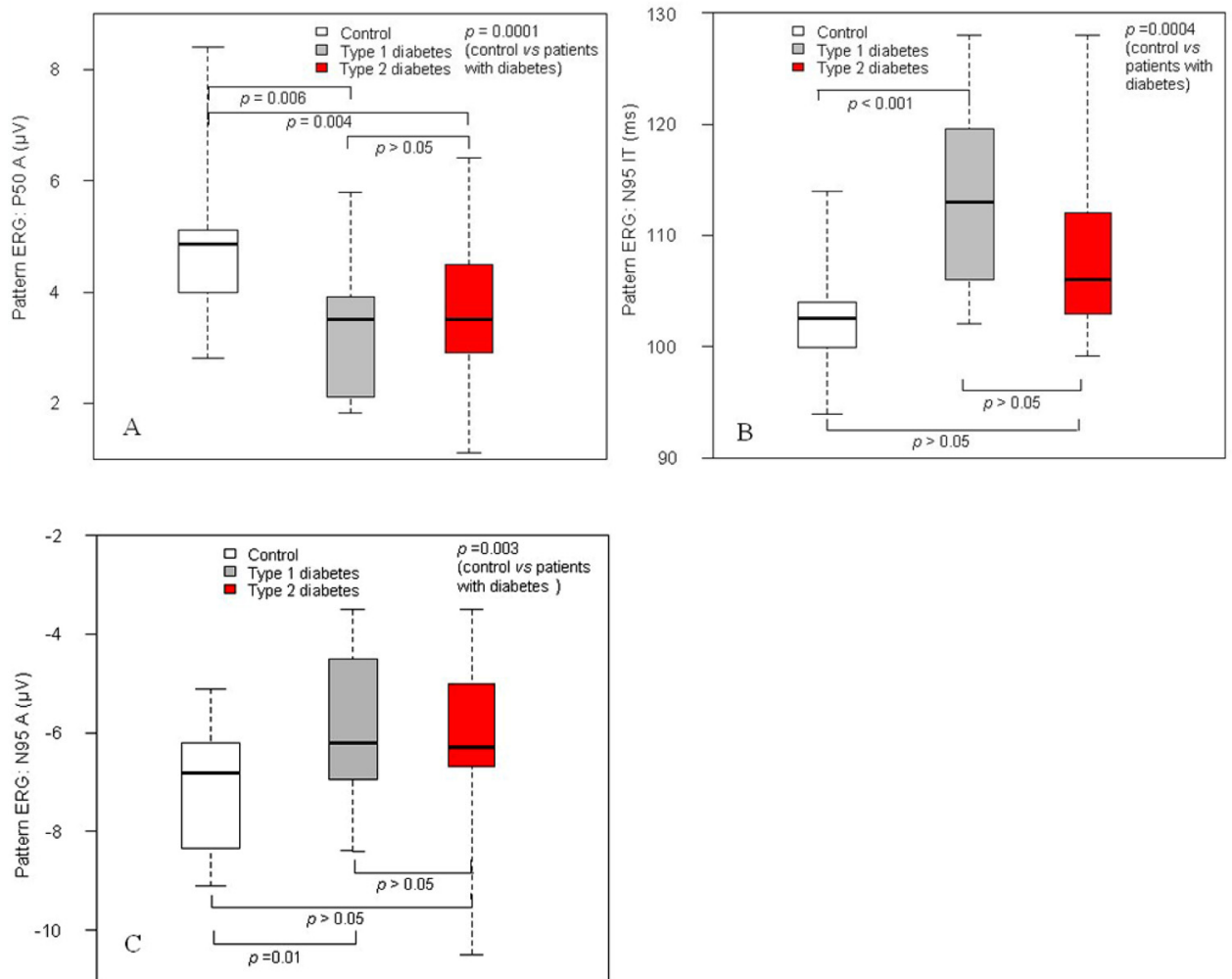

Figure 2. Pattern ERG responses in patients with diabetes and control subjects. (A) P50 amplitudes. (B) N95 implicit times. (C) N95 amplitudes. The upper and lower limits were the extremes of the distribution.

diabetes $(P<0.0001$ and $P<0.0001)$, and in patients with type 1 diabetes $(P=0.0007$ and $P=0.004)$ and type 2 diabetes $(P=0.003$ and $P<0.0001)$, compared with the controls. The sum of the amplitudes of the upward deflections was significantly reduced in patients with diabetes $(P<0.0001)$, and in those with type $1(P=0.0015)$ and type $2(P<0.0001)$, compared with the controls, but the difference between patients with type 1 and type 2 diabetes was not significant (Fig. 4).

mfERG. The average responses for the implicit times and amplitudes of N1, P1, and N2 were not significantly different in patients with diabetes and controls in any of the ring groups studied (Fig. 5)
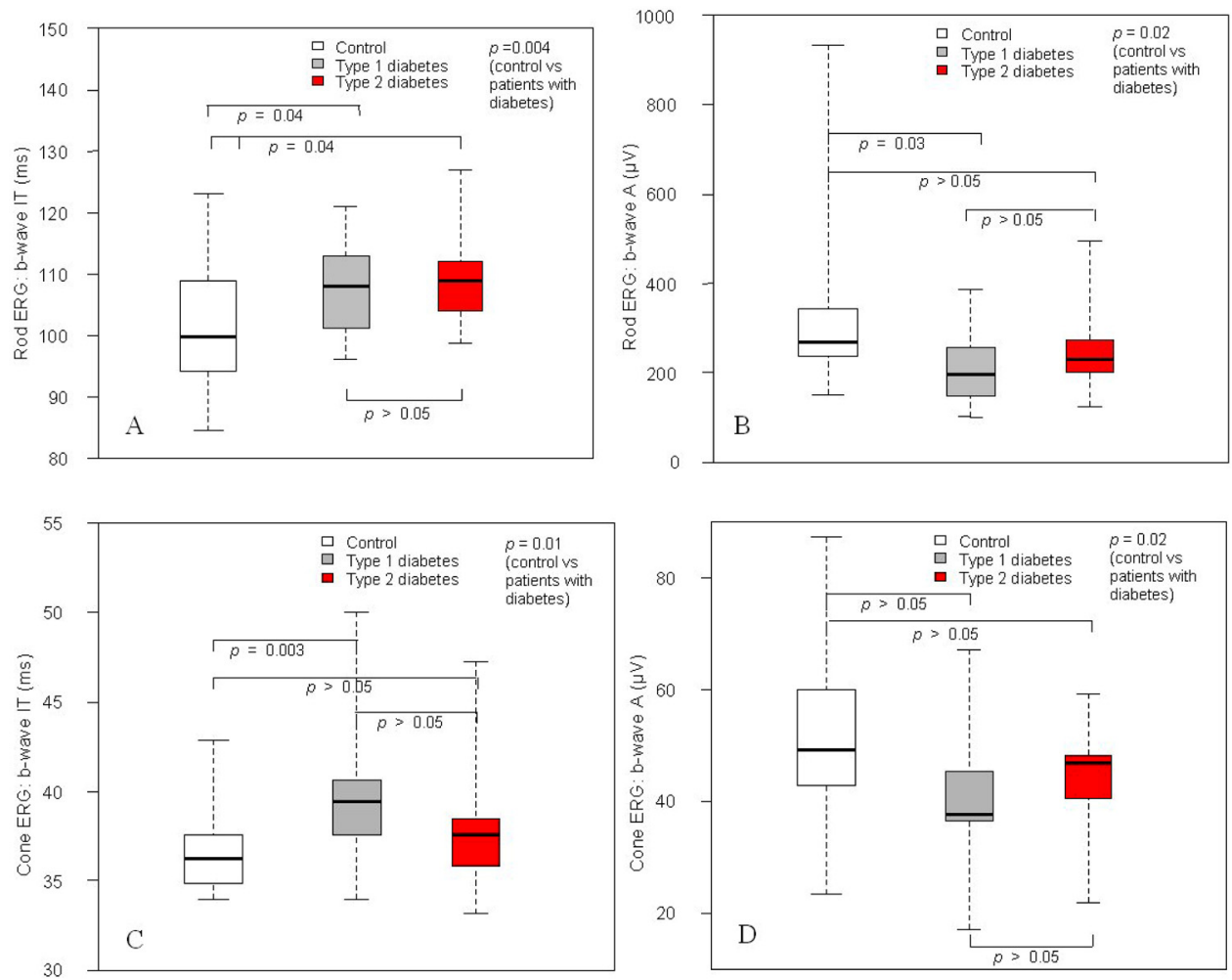

Figure 3. Full-field ERG responses in patients with diabetes and healthy controls. (A) Rod ERG: $b$-wave implicit times. (B) Rod ERG: $b$-wave amplitudes. (C) Cone ERG: $b$-wave implicit times. (D) Cone ERG: $b$-wave amplitudes. The upper and lower limits were the extremes of the distribution. 


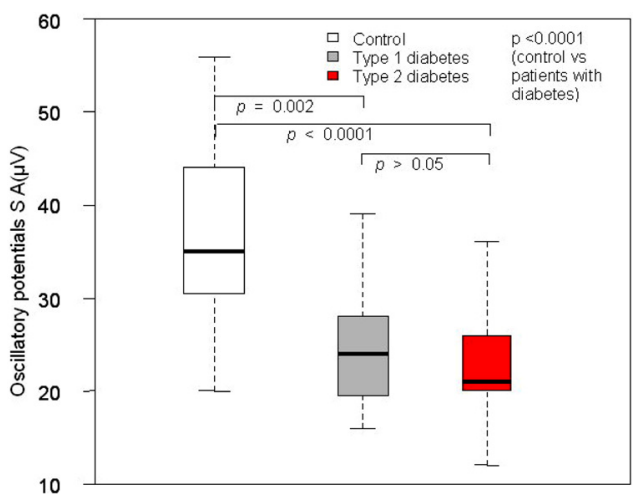

FIGURE 4. Oscillatory potential responses (sum of the amplitudes of the upward deflections) in patients with diabetes and healthy controls. The upper and lower limits are the extremes of distribution.

Correlations between Flicker-Induced Responses and ERG Responses. Among all the subjects included, correlations were found between the arterial flicker-induced response and pattern ERG responses (N95 amplitude $[r=-0.27 ; P=0.047]$ and N95 implicit time $[r=-0.35 ; P=0.008]$, rod ERG responses ( $b$-wave implicit time $[r=-0.36 ; P=0.01]$ ), and the sum of the amplitudes of the oscillatory potentials $(r=0.4$; $P=0.003)$. However, when only patients with diabetes were considered, no correlation between neural and vascular functions was found (Fig. 6).

\section{Discussion}

Neurovascular coupling is the process that enables the retina to regulate blood flow in response to neural activity. Flicker light stimulation is an original stimulus to the retina that, in healthy subjects, has been used to investigate this process because it induces increased neural activity. This increase of neural activity induced by flicker stimulation leads to retinal arterial and venous dilation ${ }^{21}$ because of the release of vasodilating factors, especially nitric oxide, from neural cells and endothelial cells. ${ }^{22-24}$ The response of the retinal vessels to flicker light can be measured noninvasively using the DVA.

Flicker-induced retinal diameter change has been shown to deteriorate early in patients with diabetes. ${ }^{3-9}$ Here we found a significant reduction of the flicker-induced response in the retinal arteries and veins of normotensive patients with diabetes with no clinically detectable DR, in agreement with the
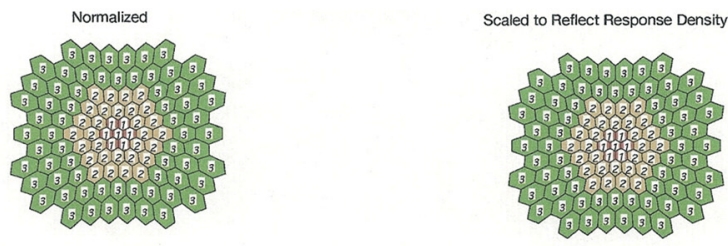

\section{Control}
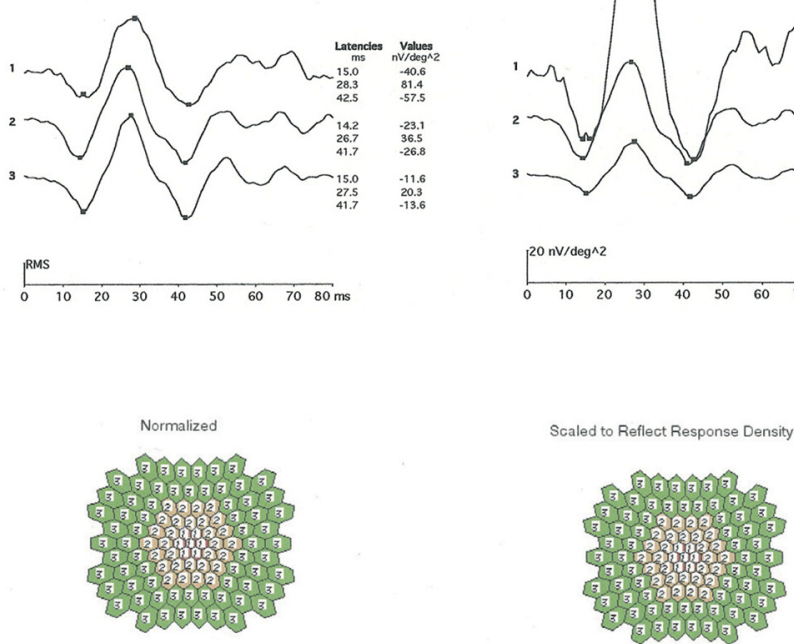
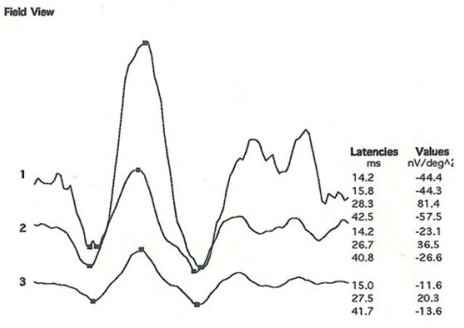

$20 \mathrm{nV} / \mathrm{deg} \wedge 2$
FIGURE 5. Example of multifocal ERG responses from a diabetes patient compared with a control subject included in the study. Both normalized and scaled responses to reflect response density are represented and summed traces for the 3 different rings are displayed. OD, right eye; OS, left eye.
Diabetes
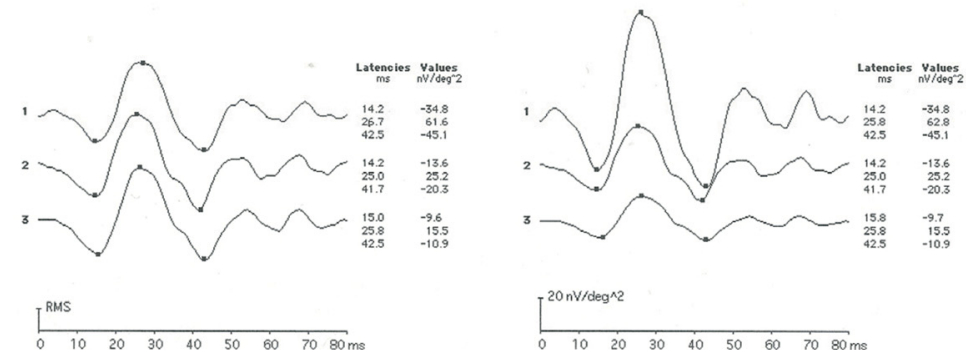

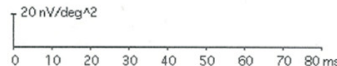



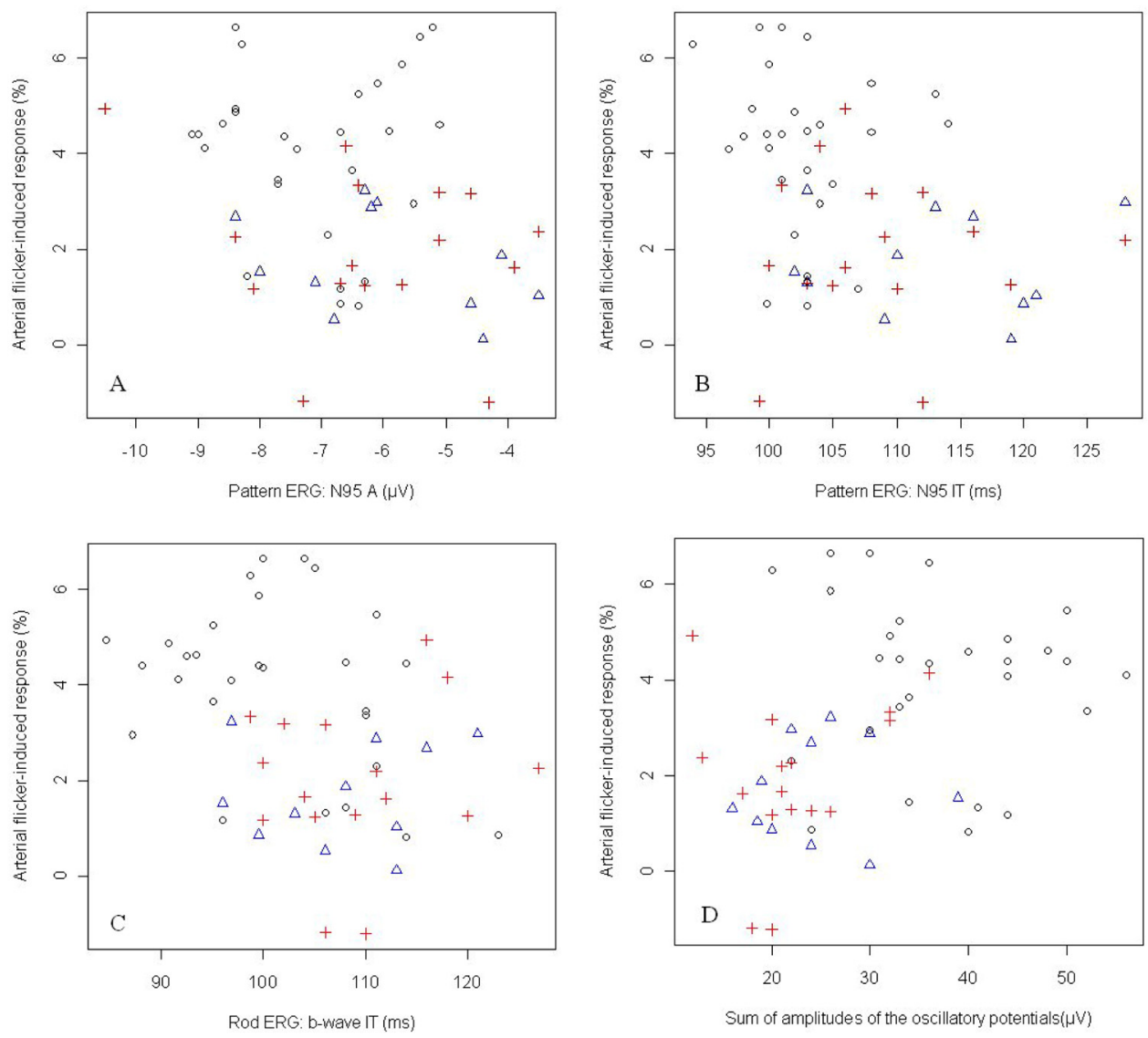

Figure 6. Correlations between the arterial flicker-induced response and ERG responses among all the subjects included, except one missing value $(N=55)$. $\bigcirc$, control subjects; $\triangle$, patients with type 1 diabetes; + , patients with type 2 diabetes. (A) Flicker-induced arterial response and N95 amplitude in pattern-ERG ( $r=$ $-0.27 ; P=0.047$ ). (B) Flicker-induced arterial response and N95 implicit time in pattern ERG $(r=$ $-0.3530 ; P=0.008)$. (C) Flicker-induced arterial response and $b$-wave implicit time in rod ERG $(r=-0.36$; $P=0.007)$. (D) Flicker-induced arterial response and the sum of the oscillatory potential amplitudes $(r=$ $0.4 ; P=0.003$ ). results of previous studies. Thus, in a recent controlled study, Mandecka et al. ${ }^{7}$ showed decreases in both arterial and venous flicker-induced retinal vasodilation in normotensive patients with type 1 diabetes and no DR. However, in a noncontrolled study of patients with type 2 diabetes with or without hypertension, Mandecka et al. ${ }^{4}$ demonstrated in these patients, after further analysis taking account of age and antihypertensive treatment, the decreases in the arterial and venous flickerinduced responses were no longer significant. Last, in a large series including 224 patients with diabetes and 103 nondiabetic control subjects, Nguyen et al. ${ }^{6}$ showed that reduced retinal vasodilation after flicker light stimulation was associated with diabetes, independently of major risk factors, including hypertension and glycemic control. However, because age and hypertension levels may influence the response to such stimulation, we included in the present study only normotensive patients with type 1 and type 2 diabetes and sex- and agematched healthy controls.

The impaired flicker-induced vascular response may be partly caused by endothelial dysfunction, as suggested by Mandecka et al. ${ }^{4,7}$ and Nguyen et al., ${ }^{6,19}$ given the documented role of nitric oxide in this flickering light-induced vasodilation..$^{3,22-24}$ However, in patients with endothelial dysfunction, Pemp et al. ${ }^{9}$ failed to demonstrate any correlation of the measurements of flowmediated vasodilatation in the forearm-the most widely used technique for the assessment of endothelial function-and flicker-induced retinal vasodilation. Impaired neurovascular coupling may thus also reflect early retinal neurodegeneration that occurs soon after the onset of diabetes. ${ }^{1,2,25}$ Recently, Luu et al. $^{26}$ demonstrated that oscillatory potential components correlate with retinal arteriolar caliber in patients with diabetes, suggesting that there is a correlation between retinal neuronal dysfunction and microvasculature changes.
The results of the retinal functional tests in our study suggest neuronal impairment in patients with diabetes, which is consistent with the impairment of the inner postreceptoral retina and, to a lesser degree, of the photoreceptor cells. We indeed found decreased amplitude and implicit time shifts in rod and cone $b$-waves and in photopic responses that originate primarily in the inner retina. The impairment of color vision, which is in agreement with previous findings, ${ }^{27,28}$ may be consistent with the impairment of the macular short-wavelength cone system, especially at a postreceptoral level. ${ }^{28,29}$

Oscillatory potential impairment was one of the earliest functional retinal abnormalities reported in the literature in patients with a short duration of diabetes, ${ }^{30}$ or no DR, ${ }^{31}$ before changes in pattern $\mathrm{ERG}^{32}$ or blood-retinal barrier permeability, ${ }^{16}$ probably indicating early deterioration of the neuronal synaptic activity of the amacrine cells. ${ }^{33}$ The results for oscillatory potentials in the present study strengthen these findings.

Both the P50 and the N95 components of pattern ERG were significantly impaired in our patients with diabetes compared with controls, suggesting the impairment of macular cone cells and retinal ganglion cells. ${ }^{34}$ The fact that pattern ERG, but not the Pelli-Robson test, which also reflects the activity of the retinal ganglion cells, was significantly impaired in the present study might have been due to the lower sensitivity of the latter test for the detection of retinal ganglion cell impairment. ${ }^{35}$

We demonstrated here that in a population of patients with diabetes and healthy controls, there is a strong overall correlation between inner retinal function and the vascular responses to flicker light. Arterial flicker-induced responses indeed correlated with the amplitude and implicit time of the N95 component of the ERG pattern, the $b$-wave implicit time of the rod ERG responses, and the oscillatory potentials. Riva et al. ${ }^{24}$ suggested that flicker light-induced vasodilation was me- 
diated primarily by ganglion cells whose function is strongly correlated with the ERG pattern. Moreover, similar impairment of neurovascular coupling during glaucoma, which primarily affects ganglion cell function, has been reported, again suggesting the involvement of ganglion cells in the flicker response. ${ }^{36}$ Therefore, the impairment of flicker-induced responses may be partly attributed to the inner retinal dysfunction that occurs early in diabetes, before any clinically detectable DR. ${ }^{1,2,25}$ However, when considering only patients with diabetes, we did not find any correlation between the flicker-induced diameter response and neural function, possibly because of a lack of power. In addition, because of the exploratory nature of the present study, our results must be interpreted with caution. Conversely, neural dysfunction and impaired flicker-induced response may constitute two different pathways of hyperglycemia complications that are not necessarily correlated.

In conclusion, we found here that in patients with diabetes, neural and neurovascular dysfunctions are early events, both of which precede the onset of clinically detectable DR. We also found that in the study group as a whole, inner retinal function correlated with the vascular responses to flicker light. However, this correlation was not found when only patients with diabetes were considered. Further investigations are needed to establish whether neurovascular dysfunction in the early stages of diabetes is linked to an inner neural dysfunction.

\section{References}

1. Barber AJ. A new view of diabetic retinopathy: a neurodegenerative disease of the eye. Prog Neuropsychopharmacol Biol Psychiatry. 2003;27:283-290.

2. Antonetti DA, Barber AJ, Bronson SK, et al. Diabetic retinopathy: seeing beyond glucose-induced microvascular disease. Diabetes. 2006;55:2401-2411.

3. Garhofer G, Zawinka C, Resch H, Kothy P, Schmetterer L, Dorner GT. Reduced response of retinal vessel diameters to flicker stimulation in patients with diabetes. Br J Ophthalmol. 2004;88:887891.

4. Mandecka A, Dawczynski J, Blum M, et al. Influence of flickering light on the retinal vessels in diabetic patients. Diabetes Care 2007;30:3048-3052.

5. Bek T, Hajari J, Jeppesen P. Interaction between flicker-induced vasodilatation and pressure autoregulation in early retinopathy of type 2 diabetes. Graefes Arch Clin Exp Ophthalmol. 2008;246: 763-769.

6. Nguyen TT, Kawasaki R, Wang JJ, et al. Flicker-light induced retinal vasodilation in diabetes and diabetic retinopathy. Diabetes Care. 2009;32:2075-2080.

7. Mandecka A, Dawczynski J, Vilser W, et al. Abnormal retinal autoregulation is detected by provoked stimulation with flicker light in well-controlled patients with type 1 diabetes without retinopathy. Diabetes Res Clin Pract. 2009;86:51-55.

8. Pemp B, Garhofer G, Weigert G, et al. Reduced retinal vessel response to flicker stimulation but not to exogenous nitric oxide in type 1 diabetes. Invest Ophthalmol Vis Sci. 2009;50:4029-4032.

9. Pemp B, Weigert G, Karl K, et al. Correlation of flicker-induced and flow-mediated vasodilatation in patients with endothelial dysfunction and healthy volunteers. Diabetes Care. 2009;32:1536-1541.

10. Nagel E, Vilser W, Lanzl I. Age, blood pressure, and vessel diameter as factors influencing the arterial retinal flicker response. Invest Ophthalmol Vis Sci. 2004;45:1486-1492.

11. Bowman KJ. A method for quantitative scoring of the Farnsworth Panel D-15. Acta Ophthalmol. 1982;60:907-916.

12. Holder GE, Brigell MG, Hawlina M, Meigen T, Vaegan, Bach M ISCEV standard for clinical pattern electroretinography: 2007 update. Doc Ophthalmol. 2007;114:111-116.

13. Marmor MF, Holder GE, Seeliger MW, Yamamoto S. Standard for clinical electroretinography (2004 update). Doc Ophthalmol. 2004;108:107-114.
14. Hood DC, Bach M, Brigell M, et al. ISCEV guidelines for clinical multifocal electroretinography (2007 edition). Doc Ophthalmol. 2008;116:1-11.

15. Yonemura D. [An electrophysiological study on activities of neuronal and non-neuronal retinal elements in man with reference to its clinical application]. Nippon Ganka Gakkai Zasshi. 1977;81: 1632-1665.

16. Yoshida A, Kojima M, Ogasawara H, Ishiko S. Oscillatory potentials and permeability of the blood-retinal barrier in noninsulin-dependent diabetic patients without retinopathy. Ophthalmology. 1991; 98:1266-1271.

17. Vilser W, Nagel E, Lanzl I. Retinal vessel analysis, new possibilities Biomed Tech (Berl.) 2002;47:682-685.

18. Nagel E, Vilser W, Fink A, Riemer T. [Variance of retinal vessel diameter response to flicker light: a methodical clinical study]. Ophthalmologe. 2006;103:114-119.

19. Nguyen T, Kawasaki R, Kreis AJ, et al. Correlation of light-flickerinduced retinal vasodilation and retinal vascular caliber measurements in diabetes. Invest Ophthalmol Vis Sci. 2009;50:5609-5613.

20. Dorner GT, Garhöfer G, Huemer KH, Riva CE, Wolzt M, Schmetterer L. Hyperglycemia affects flicker-induced vasodilation in the retina of healthy subjects. Vision Res. 2003;43:1495-1500.

21. Formaz F, Riva CE, Geiser M. Diffuse luminance flicker increases retinal vessel diameter in humans. Curr Eye Res. 1997;16:12521257.

22. Buerk DG, Riva CE, Cranstoun SD. Nitric oxide has a vasodilatory role in cat optic nerve head during flicker stimuli. Microvasc Res. 1996;52:13-26.

23. Dorner GT, Garhofer G, Kiss B, et al. Nitric oxide regulates retinal vascular tone in humans. Am J Physiol Heart Circ Physiol. 2003; 285:631-636.

24. Riva CE, Logean E, Falsini B. Visually evoked hemodynamical response and assessment of neurovascular coupling in the optic nerve and retina. Prog Retin Eye Res. 2005;24:183-215.

25. Kern TS, Barber AJ. Retinal ganglion cells in diabetes. $J$ Physiol. 2008; 586:4401- 4408 .

26. Luu CD, Szental JA, Lee SY, Lavanya R, Wong TY. Correlation between retinal oscillatory potentials and retinal vascular caliber in type 2 diabetes. Invest Ophthalmol Vis Sci. 2010;51:482- 486.

27. Hardy KJ, Fisher C, Heath P, Foster DH, Scarpello JH. Comparison of colour discrimination and electroretinography in evaluation of visual pathway dysfunction in aretinopathic IDDM patients. $\mathrm{Br} \mathrm{J}$ Ophthalmol. 1995;79:35-37.

28. Kurtenbach A, Schiefer U, Neu A, Zrenner E. Preretinopic changes in the colour vision of juvenile diabetics. Br J Ophthalmol. 1999; 83:43- 46

29. Greenstein VC, Shapiro A, Zaidi Q, Hood DC. Psychophysical evidence for post-receptoral sensitivity loss in diabetics. Invest Ophthalmol Vis Sci. 1992;33:2781-2790.

30. Holopigian K, Seiple W, Lorenzo M, Carr R. A comparison of photopic and scotopic electroretinographic changes in early diabetic retinopathy. Invest Ophthalmol Vis Sci. 1992;33:2773-2780.

31. Juen S, Kieselbach GF. Electrophysiological changes in juvenile diabetics without retinopathy. Arch Ophthalmol. 1990;108:372375 .

32. Coupland SG. A comparison of oscillatory potential and pattern electroretinogram measures in diabetic retinopathy. Doc Ophthalmol. 1987;66:207-218.

33. Wachtmeister L. Oscillatory potentials in the retina: what do they reveal. Prog Retin Eye Res. 1998;17:485-521.

34. Holder GE. Pattern electroretinography (PERG) and an integrated approach to visual pathway diagnosis. Prog Retin Eye Res. 2001; 20:531-561.

35. Stewart WC, Chauhan BC. Newer visual function tests in the evaluation of glaucoma. Surv Ophthalmol. 1995;40:119-135.

36. Riva CE, Salgarello T, Logean E, Colotto A, Galan EM, Falsini B. Flicker-evoked response measured at the optic disc rim is reduced in ocular hypertension and early glaucoma. Invest Ophthalmol Vis Sci. 2004; $45: 3662-3668$. 